\title{
Development of Cold Light Indirect Ophthalmoscopic Video System for Sharing Stereopsis
}

\author{
Hyoun-Joong Kong, Jae Pyeong Cha, Jong Mo Seo, Jeong Min Hwang, Hum Chung and Hee Chan Kim
}

\begin{abstract}
To improve the usability of conventional indirect ophthalmoscope, we propose the newly designed binocular indirect ophthalmoscope which is inexpensive, comfortable, light-weighted, long-lasting, and provides stereopsis not only to examiner but also to observers. Our new system shows good performance compared to conventional indirect ophthalmoscope and also presents the stereoscopic assistant view through special visualization system.
\end{abstract}

\section{INTRODUCTION}

$\mathrm{O}$ PHTHALMOSCOPY is an examination of the fundus, which reveals the pathologic changes in the retina, optic disc, choroid, and retinal blood vessels, thus it is performed as a part of a routine ophthalmologic examination. It is useful in detecting and evaluating various retinal diseases, such as retinal detachment, retinal holes and tears, diabetic or hypertensive retinopathy, retinal vascular disorders and so on.

Direct ophthalmoscope is light-weighted, small-sized, convenient, and portable. It provides good spatial resolution in magnified view, thus it is convenient in evaluating focal lesions around optic nerve head. However, it does not provide stereopsis and is much influenced by the transparency of cornea, lens, and vitreous.

Binocular indirect ophthalmoscope (BIO) provides inverted, large field of view, thus it is convenient to evaluate the large-sized pathological changes such as diabetic retinopathy. It also provides the stereoscopic view, and these features are really convenient to visualize the retinal detachment. However, BIO requires high degree of operator skills since it shows inverted, reversed image of retina and the proper magnifying lens should be handled simultaneously during the examination. And also it is somewhat heavy to wear on head for a long time. Because it uses a halogen bulb as a light source, it radiates too much heat and efficiency of power consumption is low. Moreover the light bulb is

Manuscript received April 16, 2007. This work was supported by Grant No. R01-2006-000-10717-0 and grant No. R01-2005-000-10875-0 from the Basic Research Program of the Korea Science \& Engineering Foundation.

Hyoun-Joong Kong and Jae Pyeong Cha with Interdisciplinary Program, Biomedical Engineering Major, Graduate School, Seoul National University, Seoul, Korea (email: gongcop@melab.snu.ac.kr)

Jong Mo Seo is with Department of Ophthalmology, Dongguk University, Seoul, Korea.(email: callme@snu.ac.kr).

Jeong Min Hwang and Hum Chung is with Department of Ophthalmology, Seoul National University Hospital, Seoul 110-744, Korea.(email: callme@snu.ac.kr).

Hee Chan Kim is with Department of Biomedical Engineering, College of Medicine and Institute of Medical and Biological Engineering, Medical Research Center, Seoul National University, Seoul, Korea (email:hckim@snu.ac.kr). expensive and has a short life time. For the observer during the indirect ophthalmoscopic examination, beam-splitting observation mirror can be attached in front of the viewing window, but it is difficult to follow the examiner's head movement during the examination. In case of the video-assisted indirect ophthalmoscope, it is much heavier than conventional one, thus it is not so commonly adopted even for the educational purpose. Recently, Spectra Plus (Keeler Limited, Windsor, UK) is released for light-weighted and easy wearing prototype. However it cannot provide the assistant view.

In this paper, the newly designed BIO is proposed, which is inexpensive, portable, comfortable, compact and light-weighted. And it also provides stereoscopic assistant view through the $3 \mathrm{D}$ viewer unit.

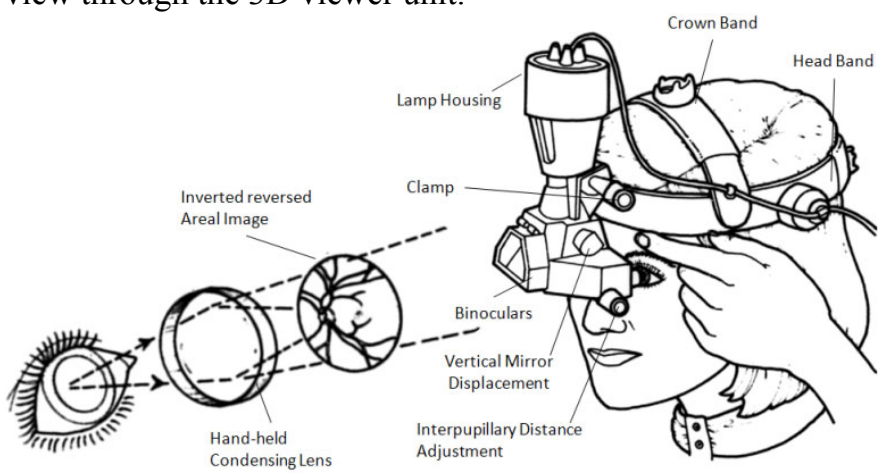

Fig. 1. Standard components of conventional binocular indirect ophthalmoscope (BIO)

\section{MAterial AND Methods}

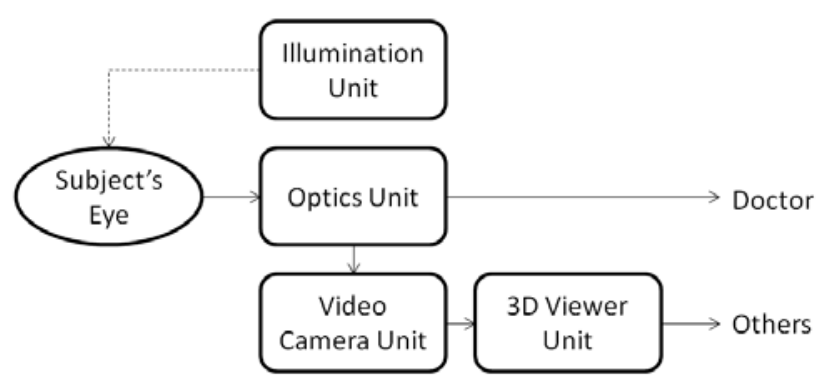

Fig. 2. Block diagram of newly devised BIO system, which is consisted of illumination, optics, video camera, $3 \mathrm{D}$ viewer units. 


\section{A. Illumination Unit}

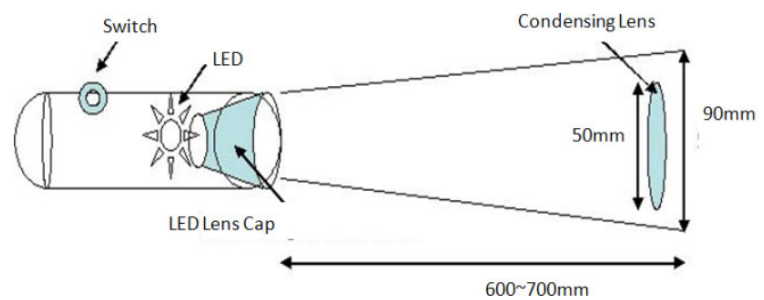

Fig. 3. Illumination Unit

As a light source, the white light emission diode (LED) with $1 \mathrm{~W}$ power is used instead of halogen bulb that is used as the illumination source of conventional indirect ophthalmoscope. The LED provides bright and homogenous light with much less heat. And its low power consumption and long durability more than 100,000 hours eliminates the need of replacement of light source.

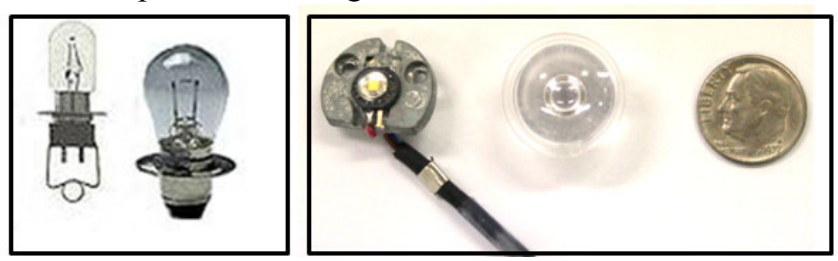

Fig. 4. Halogen bulb vs LED with lens cap

The desired angle of illumination by LED light source was acquired with the LED lens cap which focused the beam into the hand-held condensing lens fairly well in the degree of $8^{\circ}$ (Fig. 3).

Compact, 3.7V lithium ion battery (Amperex Technology Limited, Tsuen Wan, N. T., Hong Kong) was used as a power source. It can be clipped onto the waist belt when in use and charged through the USB port of personal computer. A fully charged battery lasted about 5 hours of continuous use without recharging

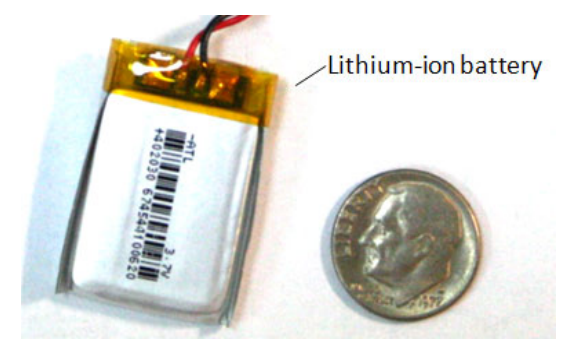

Fig. 5. Lithium ion battery (3.7V)

\section{B. Optics Unit}

The optics unit is consisted of binocular eyepieces and hand-held magnifying lens. Light from illumination unit is directed into patient's eye. Reflected beams from retina are focused through hand-held magnifying lens and inverted, reversed retinal image is produced. And this virtual image is transmitted into binocular eyepieces.

In conventional $\mathrm{BIO}$, the visual axis is completely reflected on two $45^{\circ}$-tilted mirrors and sent to examiner's eyes (Fig. 6 (a)). In the new system, the image is at fist sent to right angle prism, which reflects the visual axis by $90^{\circ}$ and after then, the axis passes the polarizing cube beam splitter, which splits randomly polarized beams into two orthogonal, linearly polarized components: S-polarized light is reflected into examiner's eye at a $90^{\circ}$ angle while P-polarized light transmitted into temporal direction, where tiny video camera sensor modules are located (Fig. 6 (b)). The ratio of transmission and reflection used in this design was 1.

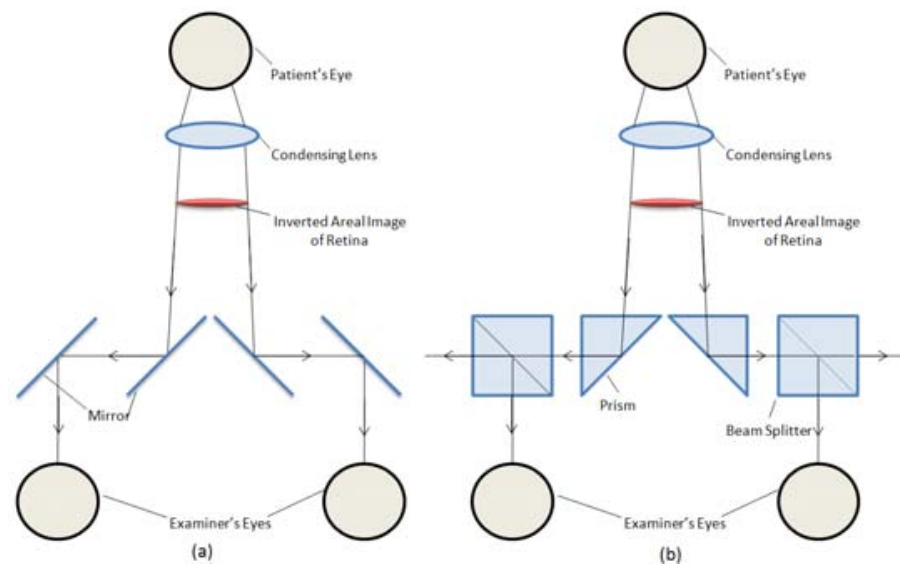

Fig. 6. Schematic representation of the conventional BIO (a) and newly designed one (b).

The interpupillary distance of the observation window in the binocular eyepiece can be adjusted between 55 and $73 \mathrm{~mm}$ according to the examiner's (Fig. 6).

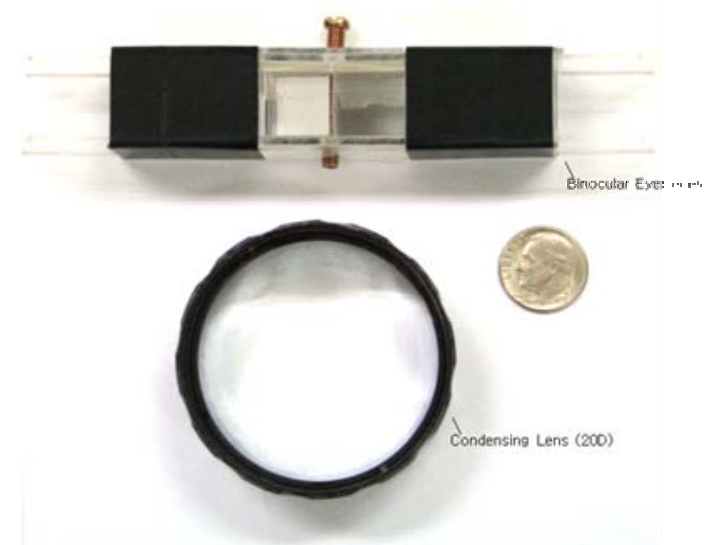

Fig. 7. Optic unit composed of binocular eyepiece and 20 diopter condensing lens

To condense the beam from illumination source and magnify reflected beams from retina, the conventional 20 diopter aspheric lens was used (Fig. 7). 


\section{Video Camera Unit}

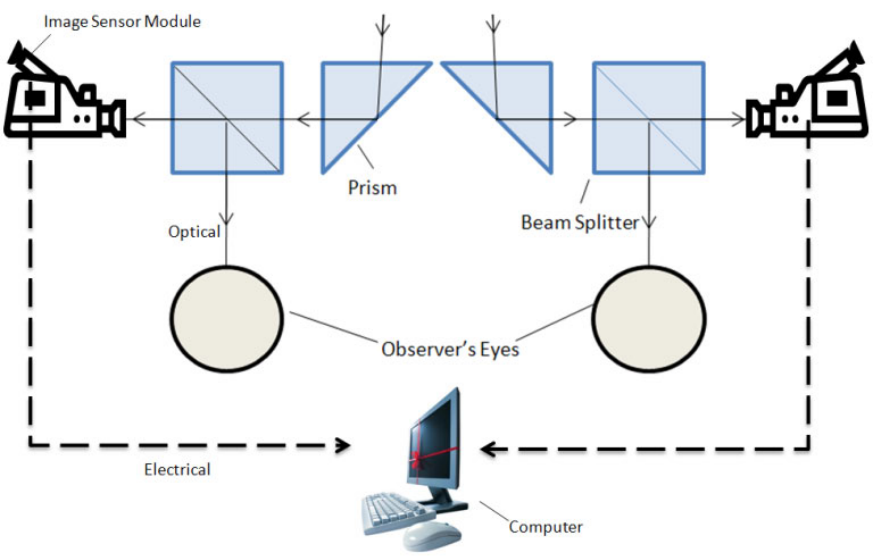

Fig. 8. Schematic of video camera unit attached to the system

Two CMOS sensor modules were mounted on the cube beam splitters as mentioned above (Fig. 8,9) and used to capture the retinal image at a rate of 30 frames per second. The images transmitted from two beam splitters are simultaneously sent to PC through USB 2.0 connectivity.

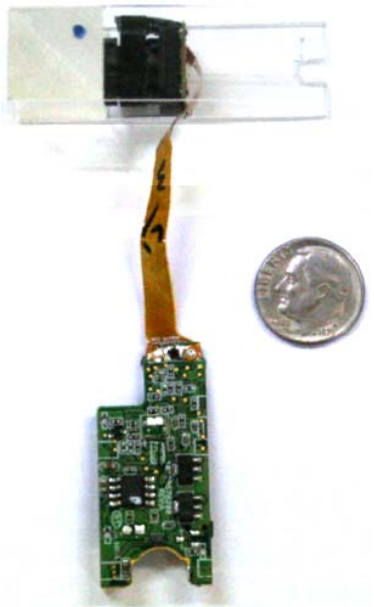

Fig. 9. CMOS sensor module attached to cube beam splitter

\section{3D Viewer Unit}

For sharing the stereopsis of $\mathrm{BIO}$, the $3 \mathrm{D}$ viewer unit was newly devised. It is consisted of frame grabbing software running on personal computer, monitor, and stereoscopic binoculars.

There is a parallax between two retinal images acquired from stereo video camera, which is a pair of image sensor modules attached to the binocular eyepiece (Fig. 8). The frame grabbing software does not only show the stereo-pair image, but also has additional features such as horizontal and vertical image flipping, automatic contrast enhancement, and saving still-shot or video data (Fig. 10). The software was developed with LabVIEW $^{\mathrm{TM}}$ v.8.2 (National Instruments, Austeen, Texas, USA).

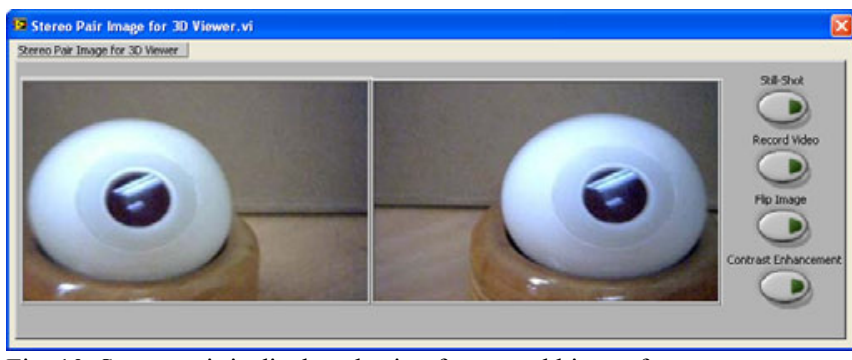

Fig. 10. Stereo-pair is displayed using frame grabbing software

Stereoscopy is a technique capable of recording 3dimensional visual information or creating the illusion of depth in an image [3]. The principle of stereoscopic 3D vision is shown in Fig. 11.

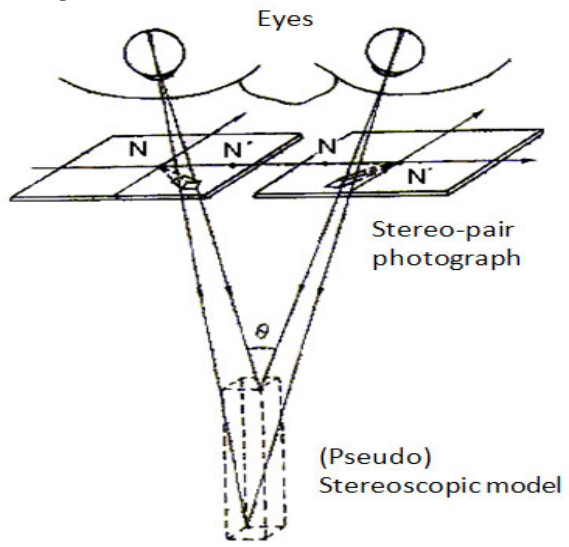

Fig. 11. Principle of stereoscopic vision

Based on the principle of stereoscopy, the stereoscope in our 3D viewer unit was made of two prisms and plastic coated card paper with the black inside surface to create the dark viewing chamber (Fig. 12). Finally, the 3D viewer unit was completed through attaching the stereoscope to the LCD monitor (Fig. 13). The stereoscopic image was successfully observed through the $3 \mathrm{D}$ viewer unit.

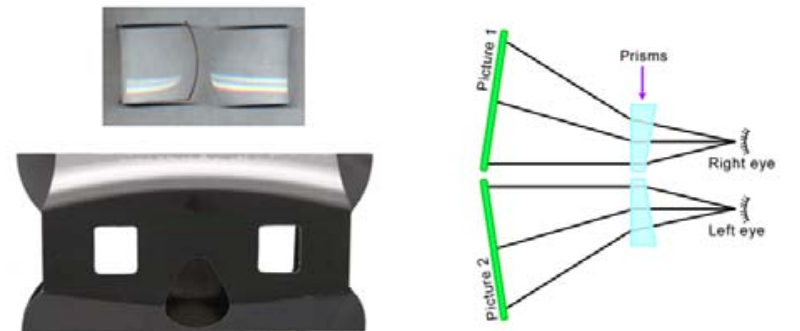

Fig. 12. Stereoscope using card paper \& prisms (left) and its principle (right). 


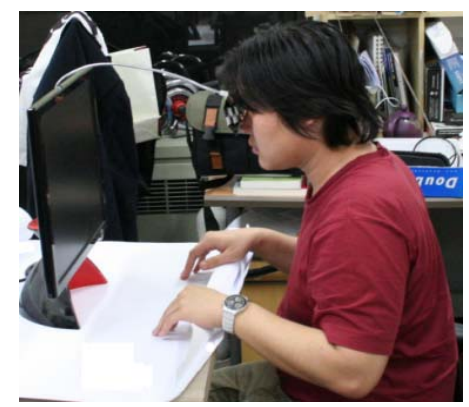

Fig. 13. The 3D viewer unit consisted of LCD monitor and stereoscope.

\section{RESULTS}

The overall indirect ophthalmoscope system integrated with the illumination, optics, video camera, and $3 \mathrm{D}$ viewer units is shown in Fig. 14. The entire optics and illumination units can be clipped on wrap-around glasses and be swung up to allow direct eye contact with the patient.

The performance of the new system was compared with the conventional $\mathrm{BIO}$ (Vantage $^{\mathrm{TM}}$, Keeler Instruments Inc. Broomall, PA, USA). The results show that our new system is superior to conventional BIO in most of the specification as shown in Table 1.

TABLE 1. PERFORMANCE COMPARISON

\begin{tabular}{lll}
\multicolumn{2}{c}{ TABLE 1. PERFORMANCE COMPARISON } \\
\hline \hline Spec. & $\begin{array}{l}\text { Conventional } \\
\text { Design }\end{array}$ & New Design \\
\hline Size & $150 \times 150 \times 60 \mathrm{~mm}$ & $90 \times 29 \times 20 \mathrm{~mm}$ \\
Weight & $500 \mathrm{~g}+850 \mathrm{~g}($ Power $)$ & $118 \mathrm{~g}+10 \mathrm{~g}($ power $)$ \\
Power & $110 \mathrm{~V}$ AC Adapter \& & $3.7 \mathrm{~V}$ lithium-ion \\
& Transformer & battery \\
& & (Rechargeable) \\
Light & Halogen Bulb & White Power LED \\
Camera & N/A & Yes \\
Heat & Hot & "Cold" light \\
Continuous Use & $\sim 3$ Hour & $\sim 35$ Hour \\
Duty Time & & \\
Wearing Type & Head-mount & Glasses-mount \\
Sharing Stereopsis & N/A & Yes \\
& & \\
\hline \hline
\end{tabular}

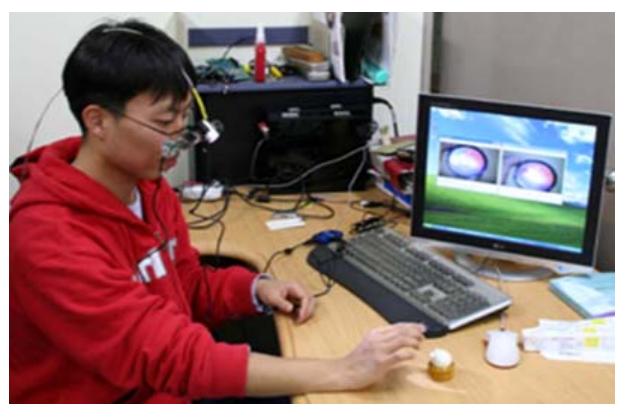

Fig. 14. Examiner wearing newly-developed BIO observes training eye model.

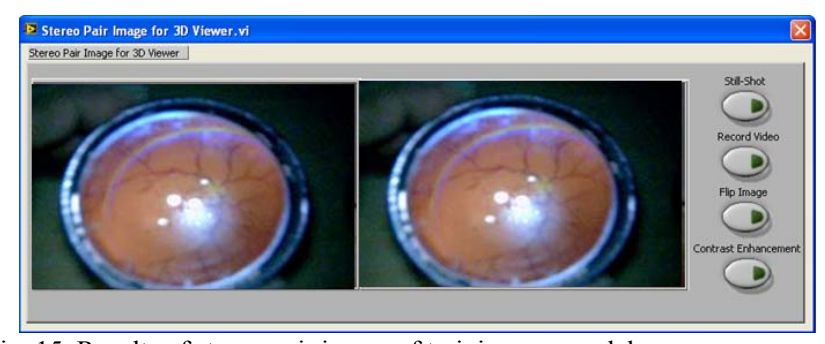

Fig. 15. Results of stereo-pair image of training eye model

For testing the integrated system, the training eye model was used. Retinal vessels, optic nerve head, and other artificial landmarks were visualized very well, and also the stereopsis of fundus image was available in the 3D viewer unit (Fig. 15).

\section{DISCUSSION}

Even though the system is successfully developed, there is something to be improved. If the type of current beam splitter in the optics unit is replaced with a plate type, total weight of the optics unit will be decreased more than $50 \%$. For convenient and exact adjustment of interpupillary distance, minute modification is still needed. In order to eliminate the flare phenomenon in the retinal image on computer monitor, polarizer or surface coating of the eyepiece may be needed. In the illumination unit, various filters (e.g. Cobalt blue and red-free filters) will be attached in front of the LED lens cap for the special use such as retinal nerve fiber layer viewing.

\section{REFERENCES}

[1] T.R. Jorgensen and D.R. Meyer, "Binocular indirect ophthalmoscopy using a standard fiberoptic surgical headlight", Ophthalmol Plast Reconstr Surg, 12(2), pp. 137-140, Jun, 1996.

[2] K.R. Rao and I.F. Wessels, "Indirect micro-ophthalmoscopy: intraoperative binocular indirect ophthalmoscopy with the operating microscope", Ophthalmic Surg., 24(6), pp. 407-410, Jun, 1993. 